\title{
Hall B superconducting magnets for the CLAS12 detector at JLAB
}

\author{
L. Quettier, V. Burkert, L. Elouadrhiri, D. Kashy, E. Leung, W. Schneider, C. Carter, J. Chen, T. \\ Juang, C. Taylor, L. Xu, B. Wagner, B. Wahrer, B. Wang, M. Wang.
}

\begin{abstract}
Hadron physics has been an essential part of the physics program with the CLAS detector in experimental hall $B$ at Thomas Jefferson National Accelerator Facility (Jefferson Lab). With the $12 \mathrm{GeV}$ upgrade of the CEBAF machine, hadron physics in Hall $B$ will be extended to a new domain of higher mass resonances and the range of higher transferred momentum using up to $11 \mathrm{GeV}$ electron beams and the upgraded CLAS12 detector. In this paper, status of the hall B superconducting magnets for the $12 \mathrm{GeV}$ upgrade is presented.
\end{abstract}

Index Terms - Detector magnet, superconducting magnet, torus coil, solenoid coil, double pancake, high magnetic field

\section{INTRODUCTION}

$\mathrm{F}$ OR new studies of quark confinement, nucleon structure, the physics of nuclei and the Standard Model's limits, Jefferson Lab has begun awarding contracts to double the $6 \mathrm{GeV}$ energy of the Continuous Electron Beam Accelerator Facility (CEBAF), upgrade its experimental halls and add a fourth hall.

The $\$ 310 \mathrm{M} 12 \mathrm{GeV}$ upgrade project, sponsored by the Department of Energy will not only double the beam energy, but also upgrade a range of existing facilities as well as adding a new building [1].

For Hall B, the upgrade consists of replacing the existing CEBAF Large Acceptance Spectrometer detector (CLAS) with a new detector system, CLAS12, which contains new magnets and detectors to capture the more forward-focused reaction products at the increased luminosity [2-3].

The forward part of CLAS12 will use a new torus coil that retains the six sector symmetry of CLAS to reuse existing detectors. Made of six very thin coils to limit obstruction of acceptance, the torus magnet is designed to allow momentum analysis for high momentum tracks from 5 to 40 degrees.

The central part of CLAS12 is based on a $5 \mathrm{~T}$ solenoid

Manuscript received August 1, 2010.

Notice: Authored by Jefferson Science Associates, LLC under U.S. DOE Contract No. DE-AC05-06OR23177. The U.S. Government retains a non exclusive, paid-up, irrevocable, world-wide license to publish or reproduce this manuscript for U.S. Government purposes.

L. Quettier, V. Burkert, L. Elouadrhiri, D. Kashy and W. Schneider are with Jefferson Lab, 12000 Jefferson Ave, Newport News, VA 23606, USA. (e-mail:quettier@jab.org).

E. Leung is with Magtec Engineering, Inc., P.O. Box 722064, San Diego, CA 92172, USA. (email: emleung1628@aol.com)

C. Carter, J. Chen, T. Juang, C. Taylor, L. Xu, B. Wagner, B. Wahrer, B. Wang and M. Wang are with WANG NMR Inc, 550 North Canyons Parkway, CA 94550, USA. (email: bert@wangnmr.com) magnet designed to allow the momentum analysis of charged particles at large angles, to create a homogeneous magnetic field region in the center for dynamically polarized target operation and to provide magnetic shielding of the particle detectors from the intense background of Moeller electrons. Finally, this magnet is actively shielded to minimize the stray field at specific locations. Design and construction of the two superconducting magnets have been awarded to Wang NMR (Livermore, CA, USA) and the upgrade is planned to be operational in 2013.

\section{FORWARD DETECTOR}

\section{A. Magnet Design}

The basic requirements for the torus magnet are driven by the physics goals and existing equipment available for the 12 $\mathrm{GeV}$ upgrade. They are summarized in Table 1. The purpose of the Torus is to provide an approximate toroidal magnetic field that bends charged particles and thus allows momentum analysis in combination with tracking detectors called drift chambers (DC). The CLAS12 detector has many detectors in close proximity (Fig. 1).

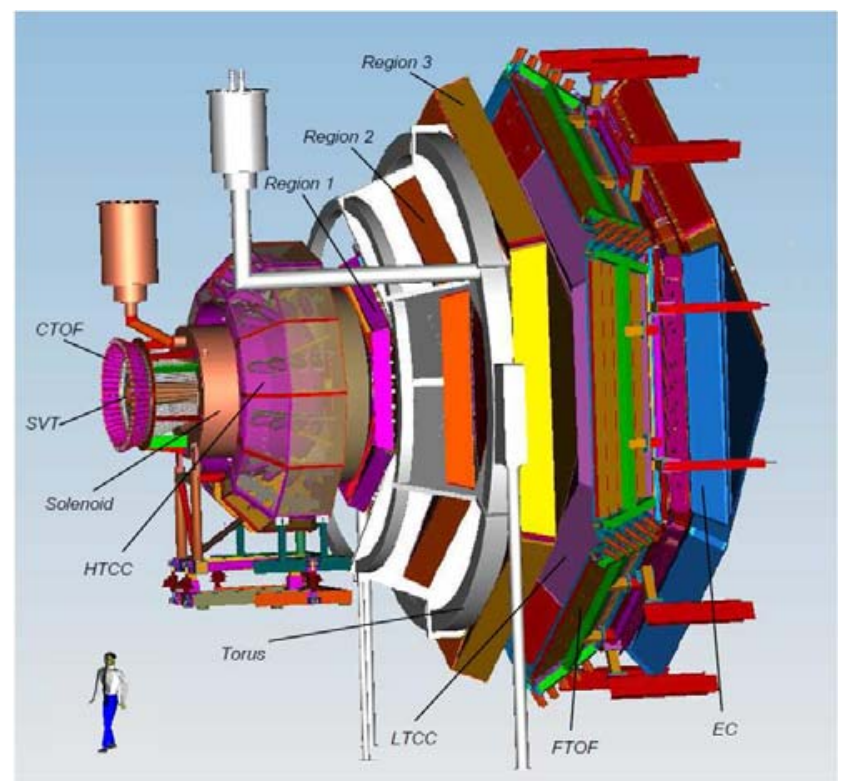

Fig. $13 \mathrm{D}$ view of the CLAS12 detector. The beam comes from the left. The target is located inside the superconducting solenoid magnet. The detector includes the electromagnetic calorimeter (EC), the high threshold Cherenkov counter (HTCC), low-threshold Cherenkov counter (LTCC), preshower calorimeter (PCAL) and central time-of-flight scintillator array (CTOF). 
The torus is required to support some of them. As such, the torus must provide the unique capability to allow precise dependable alignment. Specifically, three regions of drift chambers, an inner calorimeter (IC) and Moeller electron shielding will all be attached (Fig. 1). The torus design and support system must allow installation and removal of these detectors.

Improved electron-pion separation at higher momentum is achieved with a gas Cherenkov counter with a pion momentum threshold of $4.9 \mathrm{GeV} / \mathrm{c}$. The new high threshold Cherenkov counter (HTCC) is positioned in front of the superconducting toroidal magnet, and has to present as little material to the charged particles as practical to limit multiple scattering contributing to the momentum resolution.

The HTCC is followed by the toroidal magnet for the momentum analysis of tracks with scattering angles from $5^{\circ}$ to $40^{\circ}$. Similar to CLAS the new toroidal magnet has six superconducting coils symmetrically arranged around the beam line, and provides six sectors for charged particle detection. In each sector, tracking is accomplished with a set of 3 regions of drift chambers with 12 layers of hexagonal drift cells arranged at stereo angles of $\pm 6^{\circ}$. This arrangement provides good angular resolution both in polar angle and in azimuthal angle. The drift chamber system will provide 36 measurements for a single charged track and has sufficient redundancy for pattern recognition and track reconstruction.

TABLE I BASIC DRIVERS FOR THE TORUS MAGNET

\begin{tabular}{|c|c|c|}
\hline Parameter & Requirement & $\begin{array}{l}\text { What Drives } \\
\text { Requirement }\end{array}$ \\
\hline Number of coils & 6 & $\begin{array}{c}\text { Re-use of existing } \\
\text { detectors }\end{array}$ \\
\hline Angular coverage & $\begin{array}{c}\Theta=5^{\circ}-40^{\circ} \\
\Delta \Phi=50-90 \% \text { of } 2 \pi\end{array}$ & $\begin{array}{c}\text { Phase-space coverage from } \\
\text { exclusive reactions }\end{array}$ \\
\hline $\begin{array}{c}\int B d 1 @ \text { nominal } \\
\text { current }\end{array}$ & $\begin{array}{r}2.3 \mathrm{Tm} @ 5^{\circ} \\
0.53 \mathrm{Tm} @ 40^{\circ}\end{array}$ & $\begin{array}{c}\text { Momentum resolution for } \\
\text { charged particles } \Delta \mathrm{p} / \mathrm{p}< \\
1 \%\end{array}$ \\
\hline $\begin{array}{l}\text { Uniformity of B- } \\
\text { field in } \Phi\end{array}$ & $\begin{array}{l}\text { Limit distortions from } \\
\text { toroidal field }\end{array}$ & $\begin{array}{l}\text { Asymmetry in acceptance } \\
\text { for positive/negative tracks }\end{array}$ \\
\hline $\begin{array}{l}\text { No obstruction } \\
\text { sideways }\end{array}$ & Open access to field volume & $\begin{array}{l}\text { Access to DCs for } \\
\text { maintenance and field } \\
\text { measurement }\end{array}$ \\
\hline Coil cryostat width & $100 \mathrm{~mm}$ max & $\begin{array}{c}\text { Coverage at small angles in } \\
\text { azimuth }\end{array}$ \\
\hline Number of coils & 6 & $\begin{array}{c}\text { Re-use of existing } \\
\text { detectors }\end{array}$ \\
\hline
\end{tabular}

The torus magnet and the drift chamber system are followed by the low-threshold Cherenkov counter (LTCC) that provides charged pion identification for momenta greater than $3 \mathrm{GeV} / \mathrm{c}$. Following the LTCC are two arrays of plastic scintillators for charged particle identification. The granularity of the existing electromagnetic calorimeter (EC) will be improved by adding a preshower calorimeter of 5-6 radiation length (PCAL) in front of EC that provides a factor 2.5 better spatial resolution and a separation of two photons up to momenta greater 10
$\mathrm{GeV} / \mathrm{c}$. Coordinates used for field references are defined on figure 2 .

\section{B. Magnet design}

The torus will be made of 6 magnet coils put in separate cryostats [4]. The cryostats for each coil will be joined together to form a common vacuum space by a circular or hexagonal vacuum shell that surrounds a cold hub. The coils will be mounted on a common cold hub that will help to provide mechanical accuracy and supports magnetic and gravitational forces.

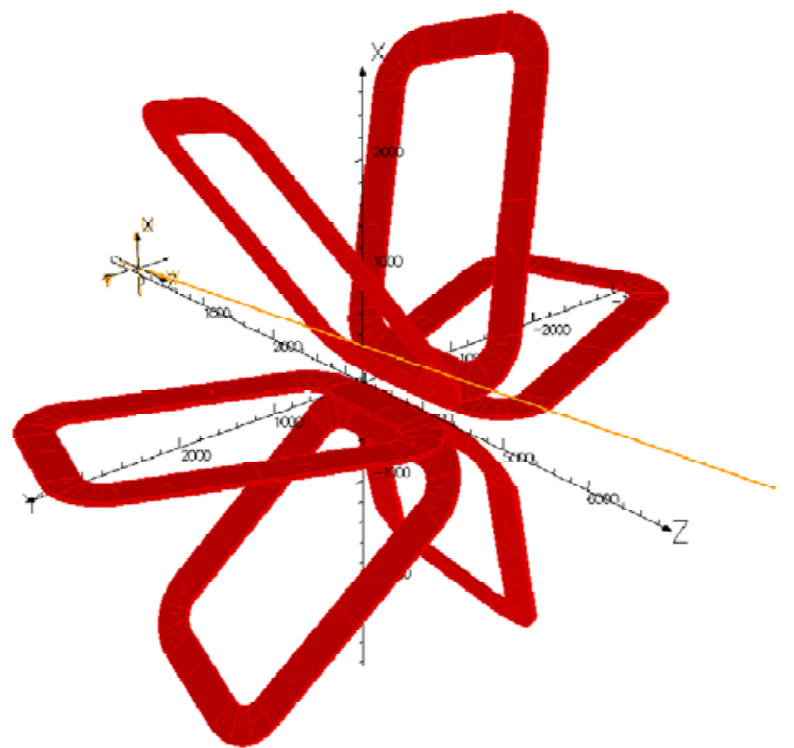

Fig. 2 Torus model showing integration line with $\mathrm{Theta}=5^{\circ}, \mathrm{Psi}=0^{\circ}$ (i.e. in $\mathrm{XZ}$ plane). Psi is a positive rotation about the $\mathrm{Z}$ axis; Theta is the angle made with the $\mathrm{Z}$ axis.

Each coil of the torus will have a trapezoidal shape and will be a double pancake made up of 124 turns. Main parameters are summarized in table II.

TABLE II TORUS MAGNET PARAMETERS

\begin{tabular}{|c|c|}
\hline Current & $3556.5 \mathrm{~A}$ \\
\hline Operating temperature & $4.5 \mathrm{~K}$ \\
\hline Bare conductor dimensions & $2.5 \mathrm{~mm} \times 20 \mathrm{~mm}$ \\
\hline Inductance & $2.24 \mathrm{H}$ \\
\hline Stored energy & $14.2 \mathrm{MJ}$ \\
\hline Peak field & $3.58 \mathrm{~T}$ \\
\hline Trapezoidal coil dimensions & $2.1 \mathrm{~m} \times(3 \mathrm{~m} \mathrm{x} \mathrm{3.5m)}$ \\
\hline Total mass & $23100 \mathrm{~kg}$ \\
\hline
\end{tabular}

As represented on figure 3, conductor is made of a SSC cable soldered in a copper channel. The conductor will have a large copper to superconductor ratio which will provide a high stability against quench. 
Critical current measurements of all the SSC spools that will be used have been performed to check the superconducting cable quality produced more than ten years ago.

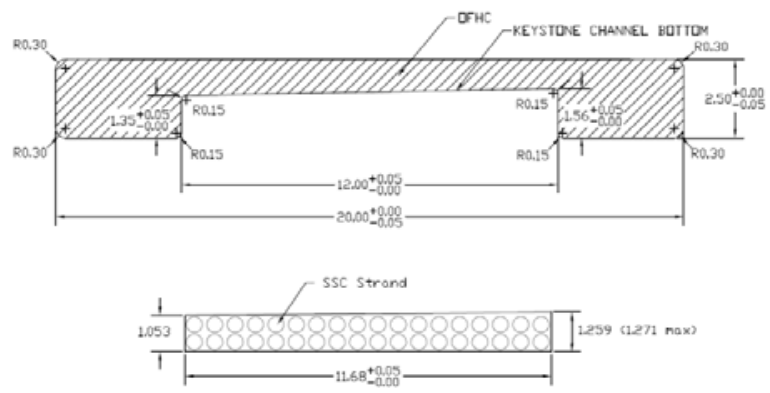

Fig. 3 Torus conductor cross-section.

Turn to turn insulation will be made of UG (Upilex-Glass), whereas layer to layer insulation will be made of GUG (GlassUpilex-Glass).

\section{Coil fabrication}

The coil winding former will be a $U$ shape coil cavity made of a 6061-T6 thick plate, machined with numerical control milling machine so that the coil formers will be made to conform to the Clas 12 Torus coil envelope with precision. The conductor will be pressed against the inner wall of the winding cavity so that the conductor, when wound, will be tightly compressed against the inner periphery. When magnet is cooled down, the copper conductor will be compressed much tighter against the coil form wall because aluminum contracts more than copper. Thus the conductor's hoop motion will be minimized because the coil pack will be compressed. Further, a stainless steel bladder will be included to provide an additional pre-stress in the coil during coil winding and during epoxy injection. The coil former will be part of the coil winding mandrel. Because of space limitation, UG turn to turn insulation would be wrapped around conductor with $100 \%$ coverage only.

\section{Magnet protection}

The stored energy will be approximately 14MJ. The magnet system will have a quench detection and protection system. In case of quench the stored energy will be dissipated in a dump resistor designed to keep the voltage below $500 \mathrm{~V}$.

\section{E. Prototype coil and schedule}

Torus design is still under study, and the final design will be completely fixed before the end of the year. In order to develop the winding procedure and to validate some of the technical choices used to design the magnet, a full size prototype coil will be manufactured. The prototype coil will be ready for warm tests by the end of 2010 .

The six coils will be delivered in June 2012 to be installed in the Hall B. Magnet assembly will take about 6 months.

\section{Central Detector}

\section{A. Physics requirements}

The Central Detectors (CD) is based on a compact solenoid magnet with maximum central magnetic field of 5T. The solenoid magnet provides momentum analysis for polar angles greater than $35^{\circ}$, protection of tracking detectors from background electrons, and acts as a polarizing field for dynamically polarized solid state targets. All three functions require high magnetic field. The overall size of the solenoid is restricted to $2.16 \mathrm{~m}$ in diameter which allows a maximum warm bore diameter of $78 \mathrm{~cm}$ for the placement of detectors.

To obtain sufficient momentum resolution in the limited space available requires high field and excellent positional resolution of the tracking detectors. The central field in the target region must also be very uniform at $\Delta \mathrm{B} / \mathrm{B}<10^{-4}$ to allow the operation of a dynamically polarized target. To achieve a sustained high polarization for polarized ammonia targets requires magnetic fields in excess of 3T. Magnetic fields of $5 \mathrm{~T}$ have been most recently used for such targets with polarization of $80 \%-90 \%$ for hydrogen [2]. In addition, the solenoidal field provides the ideal guiding field for keeping the copiously produced Moeller electrons away from the sensitive detectors and guides them to downstream locations where they can be passively absorbed in high- $Z$ material. Tracking in the $\mathrm{CD}$ is provided by a silicon vertex tracker (SVT) that uses silicon strip technology and provides tracking for polar angle from $5^{\circ}$ to $135^{\circ}$.

TABLE III BASIC DRIVERS FOR THE SOLENOID MAGNET

\begin{tabular}{|c|c|c|}
\hline Parameter & Requirement & $\begin{array}{l}\text { What Drives } \\
\text { Requirement }\end{array}$ \\
\hline Central field Bo & $5 \mathrm{~T}$ & $\begin{array}{l}\text { Particle Tracking, } \\
\text { Polarized Target }\end{array}$ \\
\hline $\mathrm{L}=1 / \mathrm{Bo} \int \mathrm{Bdl}$ & $\mathrm{L}=1$ to $1.4 \mathrm{~m}$ & $\begin{array}{l}\text { Moeller Shielding, } \\
\text { Tracking }\end{array}$ \\
\hline $\begin{array}{l}\text { Field Uniformity in } \\
\text { Target Area }\end{array}$ & $\begin{array}{c}\Delta \mathrm{B} / \mathrm{B} 0<10^{-4} \text { over a cylinder } \\
0.04 \mathrm{~m} \mathrm{~L} \times 0.025 \mathrm{~m} \varnothing\end{array}$ & Polarized target operation \\
\hline $\begin{array}{c}\text { Field at HTCC } \\
\text { PMTs }\end{array}$ & $\mathrm{B}<35$ Gauss & Stable operation of PMTs \\
\hline Full opening angle & $\Delta \Theta>41^{0}$ & Good forward tracking \\
\hline Warm bore & $\varnothing \geq 0.78 \mathrm{~m}$ & $\begin{array}{l}\text { Provide space for SVT, } \\
\text { CTOF detectors }\end{array}$ \\
\hline $\begin{array}{c}\text { Outer } \\
\text { dimensions }\end{array}$ & $\begin{aligned} \varnothing & \leq 2.16 \mathrm{~m} \\
\mathrm{~L} & \leq 1.8 \mathrm{~m}\end{aligned}$ & $\begin{array}{c}\text { For HTCC PMT, CTOF \& } \\
\text { other detector interface }\end{array}$ \\
\hline
\end{tabular}

The central time-of-flight scintillator array (CTOF) consists of 50 strips of fast plastic scintillator equipped with 100 photomultipliers that provide 2-sided light readout. The scintillator light is brought to an area of reduced magnetic field where either PMTs with lower magnetic field sensitivity can be used or where passive shielding can be employed. R\&D work is still underway to study these options. Table III 
summarizes the basic drivers for the solenoid magnet of the CLAS12 detector.

\section{B. Magnetic configuration}

The solenoid magnet will be made of 18 modules [5]. Each module will be made of two double pancakes. Central field homogeneity is obtained by adjusting the inner and outer diameter of each module (Fig. 4).

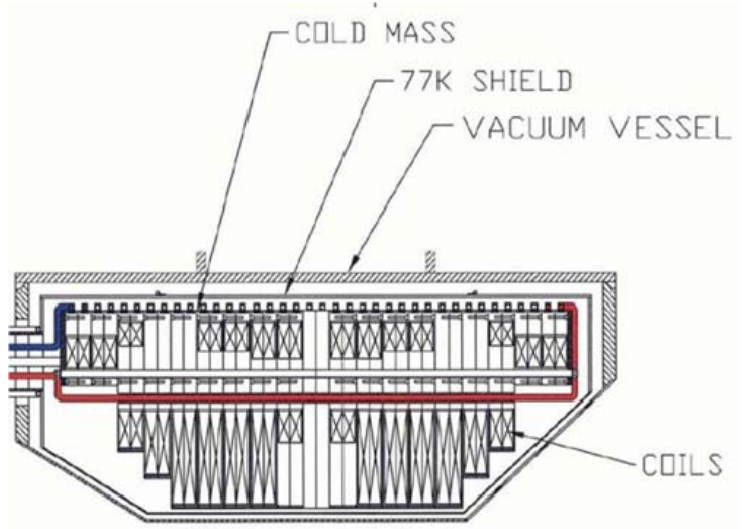

Fig. 4 Solenoid magnet cross-section.

The magnet is actively shielded to minimize the stray field at PMT locations. Besides, the same continuous length of conductor is used to avoid superconducting joints inside a coil modulus.

TABLE IV SOLENOID MAGNET PARAMETERS

\begin{tabular}{|c|c|}
\hline Current & $2434 \mathrm{~A}$ \\
\hline Number of turns & 1680 (shield) / 3992 (main) \\
\hline Operating temperature & $4.5 \mathrm{~K}$ \\
\hline Bare conductor dimensions & $2.5 \mathrm{~mm} \times 17 \mathrm{~mm}$ \\
\hline Inductance & $6.77 \mathrm{H}$ \\
\hline Stored energy & $20.06 \mathrm{MJ}$ \\
\hline Peak field & $6.74 \mathrm{~T}$ \\
\hline Total cold mass & $19300 \mathrm{~kg}$ \\
\hline
\end{tabular}

The solenoid magnet will have several common points with the torus. The solenoid conductor will also reuse SSC cable, but with a different copper channel width. The soldering line for both conductors is currently under development at Wang NMR and the conductor production will start in a near future. The coil should be wound on a coil case CNC machined from a plate approximate 2 inches thick with a $\mathrm{U}$ shaped cavity. The total length of conductor will be divided into four pancake coils. The first two pancakes will be on one coil case and then a third and fourth pancake will be wound on the second coil case. Then the two coil cases will be clamped together before tack welding and sealing with RTV silicone before pump down and epoxy vacuum impregnation.

\section{Magnet protection}

As like the torus, insulation will be made of UG (turn to turn) and GUG (ground insulation). A heater system will be incorporated to assist in spreading the quench throughout the remaining coils, thereby distributing the energy and preventing a local "hot spot" from forming. This will be incorporated into the coil supports to spread the heat from the quenching coil through the magnet. An active quench detection system will be implemented to continuously monitor a number of critical voltages from within the magnet. In case of quench, it will shut down the power supply and discharge the magnet into a dump circuit.

\section{Prototype coil and schedule}

Solenoid design is still under study, and the final design will be completely fixed before the end of the year. A prototype coil will be built for practicing ground plane insulation installation, for winding a double pancake coil, for incorporating a hoop force support SS bladder, for epoxy vacuum impregnation, for making conductor splices, installing coil cooling and for practicing coil assembly. Because it has to be typical from the winding configuration, the prototype module will contain both main coil and shield coil with coil entrance and exit terminals. The prototype will be constructed in the next few months, and will be tested at room temperature with a careful attention to the manufacturing and assembly tolerances. The completed solenoid installation at Jefferson Lab is planned for July 2012.

\section{CONCLUSION}

For Hall $\mathrm{B}$, the $12 \mathrm{GeV}$ upgrade consists of replacing the existing detector with a new detector system, which contains new magnets and detectors. The construction of the two new superconducting magnets for Hall $\mathrm{B}$ has been awarded to Wang NMR and both designs are under study. Conductor fabrication will start in the next few weeks. Final designs and prototypes should be completed by the end of 2010 .

\section{REFERENCES}

[1] C.H. Rode, "Jefferson Lab $12 \mathrm{GeV}$ CEBAF upgrade", Advances in Cryogenic Engineering 55A, edited by U.B. Balachandran et al., New York, 2009, to be published.

[2] V. Burker, "CLAS12 and its Science Program at the Jefferson Lab Upgrade", http://arXiv.org/pdf/0810.4718.

[3] S. Stepayan, "Hadron Physics with CLAS12", 13th International Conference on Hadron Spectroscopy, Tallahassee, FL, 2009, http://arXiv.org/pdf/1004.0168.

[4] B. Wang et al., "Preliminary design of JLab CLAS12 large superconducting six coil torus magnets", to be presented at this conference.

[5] B. Wang et al., "Design of a large 5T superconducting magnet for polarized target for JLAB Hall B $12 \mathrm{GeV}$ upgrade CLAS12 Detector", to be presented at this conference. 